\title{
Crisis hipercalcémica por hiperparatiroidismo primario resistente al tratamiento médico
}

\author{
Hypercalcemic crisis due to primary hyperparathyroidism resistant to medical treatment
}

\author{
Francisco Villalba-Ferrer ${ }^{1,2 *}$, Guillermo Valderas-Cortés ${ }^{1}$, Carla Basés-Valenzuela ${ }^{1}$, \\ Gara Alcalá-García-del Río ${ }^{1}$, Ana Villalba-Segarra² y Cristóbal Zaragoza Fernández \\ ${ }^{1}$ Servicio de Cirugía General y Digestiva, Consorcio Hospital General y Universitario de Valencia; ${ }^{2}$ Facultad de Ciencias de la Salud, Universidad \\ CEU Cardenal Herrera de Valencia. Valencia, España
}

\begin{abstract}
Resumen
Los pacientes con crisis hipecalcémica suelen estabilizarse con tratamiento médico y rara vez precisan tratamiento quirúrgico urgente. Se presenta el caso de una mujer con clínica inespecífica de dolor abdominal, diagnosticada tardíamente de crisis hipercalcémica por hiperparatiroidismo primario. El tratamiento médico y la diálisis peritoneal no fueron efectivos, por lo que precisó paratiroidectomía urgente con determinación intraoperatoria de hormona paratiroidea. La localización preoperatoria del adenoma fue concordante para gammagrafía y ecografía. La crisis hipercalcémica por hiperparatiroidismo primario sin respuesta al tratamiento médico precisa paratiroidectomía urgente. El diagnóstico de localización preoperatorio y la hormona paratiroidea intraoperatoria son indispensables para un tratamiento quirúrgico curativo.
\end{abstract}

Palabras clave: Adenoma de paratiroides. Hipercalcemia aguda. Hiperparatiroidismo primario.

\begin{abstract}
Patients with hypecalcemic crisis usually stabilize with medical treatment and rarely require urgent surgical treatment. Woman with a nonspecific clinic of abdominal pain, diagnosed late of hypercalcemic crisis due to primary hyperparathyroidism. Medical treatment and peritoneal dialysis were not effective, requiring urgent parathyroidectomy with determination of intraoperative parathormone. The preoperative location of the adenoma was concordant for scintigraphy and ultrasound. Hypercalcemic crisis due to primary hyperparathyroidism without response to medical treatment requires urgent parathyroidectomy. Preoperative adenoma location and intraoperative parathormone are essential for curative surgical treatment.
\end{abstract}

Key words: Parathyroid adenoma. Acute hypercalcemia. Primary hyperparathyroidism.

\section{Antecedentes}

La crisis hipercalcémica en el hiperparatiroidismo primario (HPTP) es poco frecuente (1.6-6.7\%) y se caracteriza por presentar valores muy elevados de hormona paratiroidea (PTH), que suelen superar hasta 10 veces el rango normal, y cifras de calcio sérico $>14 \mathrm{mg} / \mathrm{dl}^{1,2}$.

\footnotetext{
Correspondencia:

*Francisco Villalba-Ferrer

Dr. Sanchis Sivera, 16-12

C.P. 46008 , Valencia, España

Fecha de recepción: 04-02-2020

Cir Cir. 2020;88(S2):13-17

E-mail: flvferrer@ hotmail.com

Fecha de aceptación: 24-05-2020

DOI: $10.24875 / C I R U .20000069$

Contents available at PubMed

www.cirugiaycirujanos.com

0009-7411/@ 2020 Academia Mexicana de Cirugía. Publicado por Permanyer. Este es un artículo open access bajo la licencia CC BY-NC-ND (http://creativecommons.org/licenses/by-nc-nd/4.0/).
} 
Generalmente los pacientes pueden estabilizarse con tratamiento médico y recurrir al tratamiento quirúrgico definitivo de forma programada ${ }^{3}$.

Durante un periodo de 5 años (marzo 2015-2020) se intervinieron 35 pacientes con diagnóstico de HPTP; todos ellos presentaron un cuadro crónico, excepto un paciente que precisó tratamiento quirúrgico urgente.

Presentamos un caso clínico de crisis hipercalcémica en un HPTP sin respuesta al tratamiento médico, que precisó cirugía urgente sin poder estabilizar previamente la calcemia. Comentamos los aspectos más relevantes dada la rareza del caso presentado.

\section{Caso clínico}

Mujer de 40 años con antecedentes de hipertensión arterial y depresión, valorada en urgencias y dada de alta por dolor abdominal inespecífico. Ingresó a los 5 días por persistencia y empeoramiento del dolor abdominal, asociando náuseas, vómitos y estreñimiento.

A la exploración presentaba temblor, ansiedad, taquicardia y dolor en el hipocondrio y la fosa renal izquierda.

En la analítica al ingreso (Tabla 1 y Fig. 1) destacó un calcio corregido de $19.2 \mathrm{mg} / \mathrm{dl}$ y una PTH de $1503 \mathrm{pg} / \mathrm{ml}$, con alteración del ionograma y de la función renal.

La ecografía tiroidea detectó un nódulo hipoecoico de $3 \times 3.5 \mathrm{~cm}$ en el polo inferior del lóbulo tiroideo derecho (Fig. 2).

Se inició tratamiento con solución salina al $0.9 \%$ $(4000 \mathrm{ml} / 24 \mathrm{~h})$ y furosemida $(20 \mathrm{mg} / 8 \mathrm{~h})$. Concomitantemente se administraron calcitonina (100 Ul/8 h), cinacalcet hidrocloruro $(30 \mathrm{mg} / 12 \mathrm{~h})$ y metilprednisoIona (40 g/12 h). A las 24 horas se indicó hemodiálisis por persistencia de la hipercalcemia, bradipsiquia y alteraciones en el electrocardiograma (acortamiento de del QT, onda T aplanada).

Durante el ingreso, de forma urgente, se completó el estudio con las siguientes exploraciones:

- Gammagrafía (18mCi de 99mTc - MIBI) (Fig. 3).

- Analítica: catecolaminas libres en orina de 24 horas (cromatografía líquida de alta resolución, Kits Cromosystem ${ }^{\circledR}$ ), noradrenalina $33 \mu \mathrm{g} / 24 \mathrm{~h}$ (0-97), adrenalina $<10 \mu \mathrm{g} / 24 \mathrm{~h}(0-27)(<10)$, dopamina $430 \mu \mathrm{g} / 24 \mathrm{~h}(0-500)$. Metanefrinas fraccionadas en orina de 24 horas (cromatografía líquida de alta resolución, Kits Cromosystem $\left.{ }^{\circledR}\right)$, normetanefrinas $0.1 \mathrm{mg} / 24 \mathrm{~h}(0-0.49)$, metanefrinas 0.225 $\mathrm{mg} / 24 \mathrm{~h}(0.02-0.345), 3-m e t o x i t i r a m i n a ~ 150 \mu \mathrm{g} / 24 \mathrm{~h}$

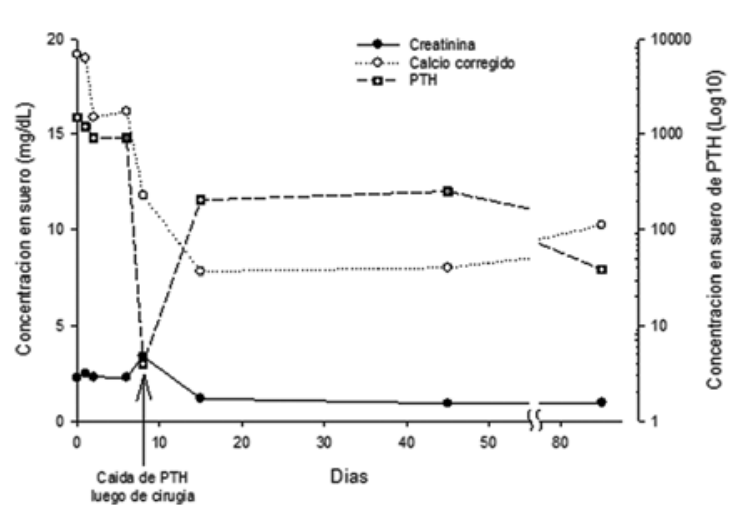

Figura 1. Gráfica de los valores de calcio, creatinina y hormona paratiroidea durante los días de hospitalización.

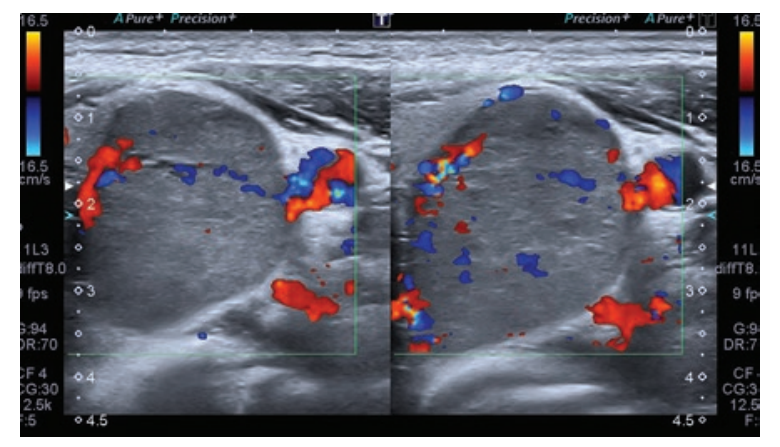

Figura 2. Ecografía cervical en la que se detecta un nódulo hipoecoico, bien delimitado, de $3 \times 3.5 \mathrm{~cm}$, con vascularización intranodular y perinodular, en el polo inferior del lóbulo tiroideo derecho, sugestivo de adenoma de paratiroides.

(100-300). Calcitonina en sangre (quimioluminiscencia, Emulite Siemens $\left.{ }^{\circledR}\right), 3.5 \mathrm{pg} / \mathrm{ml}$ (2-11.5).

- Tomografía computarizada toracoabdominal: defecto de repleción de las arterias pulmonares lobares inferiores derecha e izquierda con extensión a arterias segmentarias en relación con probable tromboembolia pulmonar aguda asintomática, sin otros hallazgos.

- Punción-aspiración con aguja fina: adenoma/hiperplasia de paratiroides y ausencia de atipias.

Tras el diagnóstico de tromboembolia pulmonar se inició anticoagulación con heparina sódica intravenosa, ajustada según los tiempos de coagulación. La heparina sódica se retiró al sexto día previo a realizar la cirugía urgente, puesto que se mantenía la hipercalcemia (16.2 mg/dl) a pesar del tratamiento médico y la hemodiálisis. Se realizaron hemitiroidectomía y paratiroidectomía izquierda en bloque, con determinación de PTH intraoperatoria (Tabla 1 y Fig. 1) y 
Tabla 1. Valores evolutivos de la analítica

\begin{tabular}{|c|c|c|c|c|c|c|c|c|}
\hline & Día 0 & Día 1 & Día 2 & Día 6 & Día 8 & Día 15 & Día 45 & Día 90 \\
\hline & Ingreso & Hemodiálisis & & Cirugía & & Alta & & \\
\hline $\begin{array}{l}\text { Creatinina, mg/dl } \\
(0.66-1.09)\end{array}$ & 2.26 & 2.50 & 2.3 & 2.29 & 3.4 & 1.17 & 0.92 & 0.96 \\
\hline $\begin{array}{l}\text { Urea, mg/dl } \\
(17.0-43.0)\end{array}$ & 71.1 & 85.6 & 56.4 & 86.8 & 130 & 58.1 & 53.8 & 26.8 \\
\hline $\begin{array}{l}\mathrm{Na}^{+}, \mathrm{mEq} / \mathrm{l} \\
(136.0-146.0)\end{array}$ & 130 & 134 & 134 & 139 & 132 & 140 & 141 & \\
\hline $\begin{array}{l}\mathrm{K}^{+}, \mathrm{mEq} / \mathrm{l} \\
(3.5-5.1)\end{array}$ & 3.1 & 3.2 & 3.2 & 4.7 & 3.5 & 5 & 4.4 & \\
\hline $\begin{array}{l}\mathrm{Cl}^{-}, \mathrm{mEq} / \mathrm{l} \\
(98.0-106.0)\end{array}$ & 92 & 99 & 99 & 107 & 103 & 111 & 106 & \\
\hline $\begin{array}{l}\text { Calcio corregido, } \mathrm{mg} / \mathrm{dl} \\
(8.8-10.6)\end{array}$ & 19.2 & 19 & 15.9 & 16.2 & 11.8 & 7.8 & 8.0 & 10.25 \\
\hline $\begin{array}{l}\text { PTH, pg/ml } \\
(12-88)\end{array}$ & 1503 & 1203 & 919 & & 4 & 205 & 252 & 38 \\
\hline $\begin{array}{l}\text { PTH intraoperatoria } \\
\text { preexéresis, pg/ml }\end{array}$ & & & & 1699 & & & & \\
\hline $\begin{array}{l}\text { PTH intraoperatoria } \\
\text { posexéresis, pg/ml }\end{array}$ & & & & 207 & & & & \\
\hline
\end{tabular}

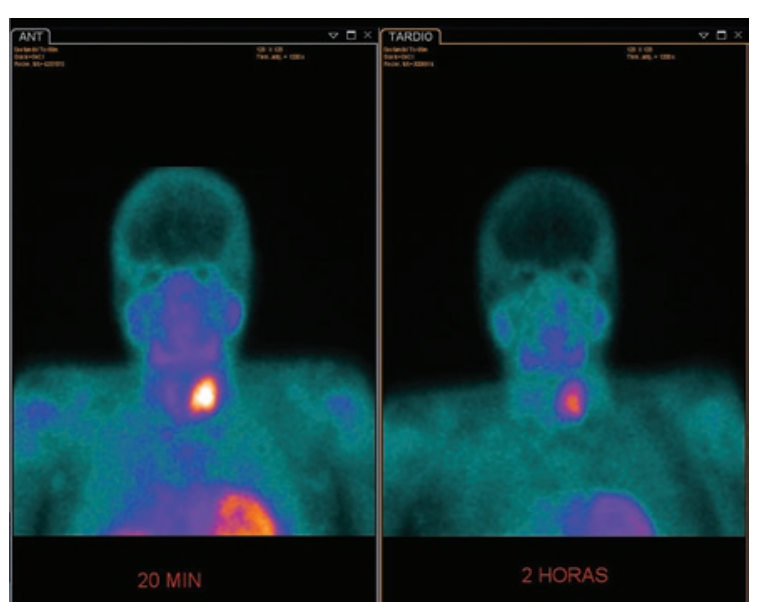

Figura 3. En la gammagrafía se detecta la persistencia de captación en el lóbulo tiroideo izquierdo a las 2 horas de la administración intravenosa de $18 \mathrm{mCi}$ de $99 \mathrm{mTc}$ - MIBI, concordante con la localización ecográfica y sugestiva de adenoma de paratiroides.

estudio anatomopatológico intraoperatorio que descartó carcinoma.

El estudio anatomopatológico diferido fue de adenoma paratiroideo de $3 \times 3 \mathrm{~cm}$ y tiroides $\sin$ lesiones.

En el posoperatorio se inició tratamiento con heparina de bajo peso molecular y calcio oral e intravenoso en función de las analíticas. Progresivamente se normalizaron la PTH, el calcio y la función renal (Tabla 1). La paciente fue dada de alta a los 15 días con calcio oral, calcitriol y prednisona, que se retiraron progresivamente. A los 6 meses está asintomática, remitió el cuadro depresivo y solo mantiene tratamiento para la hipertensión arterial.

\section{Discusión}

La forma de presentación de la crisis hipercalcémica con síntomas inespecíficos puede enmascarar el diagnóstico y retrasar su tratamiento, lo que puede suponer un desenlace fatal con evolución a una encefalopatía metabólica, insuficiencia renal y arritmia cardiaca $^{1,3}$.

Un tratamiento precoz de la hipercalcemia mejora el pronóstico ${ }^{2,3}$, pero la paciente descrita se presentó con un dolor abdominal inespecífico y un retraso diagnóstico, por lo que pensamos que el tratamiento médico iniciado de forma tardía pudo ser la causa de su mala respuesta.

En la crisis hipercalcémica por HPTP las concentraciones séricas de calcio pueden ser muy elevadas; sin embargo, hay pacientes con valores muy altos de PTH que pueden evolucionar como un HPTP crónico ${ }^{4}$ 
sin desencadenar la crisis hipercalcémica, lo que no ocurrió en el caso descrito.

Determinados tumores pueden secretar polipéptidos con estructura similar a la $\mathrm{PTH}^{5}$, y además el HPTP puede estar asociado a síndromes hereditarios de neoplasia endocrina múltiple (MEN) ${ }^{6}$. En el MEN I (HPTP, tumores de hipófisis anterior y gastroenteropáticos), el HPTP es la afectación más frecuente (95\%) y es multiglandular, pero las glándulas pueden no afectarse todas en el mismo momento, por lo que el diagnóstico de MEN I puede retrasarse al completar el estudio tras la nueva hipercalcemia. En el MEN 2a (carcinoma medular de tiroides, feocromocitoma, HPTP), el HPTP es la manifestación menos frecuente (20-30\%), la hipercalcemia suele ser leve y normalmente no afecta a las cuatro glándulas ${ }^{6}$.

En el caso descrito se localizó un adenoma único en el polo inferior del lóbulo tiroideo derecho y se completó el estudio (catecolaminas, metanefrinas, calcitonina, tomografía computarizada) sin evidenciar otro componente glandular ni alteraciones analíticas que hicieran sospechar carcinoma medular de tiroides ni feocromocitoma en el momento del diagnóstico. Se diagnosticó HPTP esporádico, hecho que no descarta que posteriormente se pueda presentar otra asociación.

Las hipercalcemias graves con cifras muy elevadas de PTH se han descrito asociadas al carcinoma de paratiroides ${ }^{7}$, por lo que se realizó el estudio citológico mediante punción-aspiración con aguja fina, que en la paciente fue negativo para células malignas.

El manejo de la crisis hipercalcémica por HPTP es inicialmente una emergencia que requiere tratamiento médico y luego un abordaje quirúrgico $0^{1,3,8}$. La rehidratación y la administración de diuréticos de asa para aumentar la excreción de calcio, junto con los bisfosfonatos y la calcitonina que inhiben la resorción ósea, son la piedra angular del manejo inicial de la hipercalcemia'. Otros tratamientos, como el cinacalcet para disminuir la concentración de PTH y los corticosteroides para disminuir el calcitriol, también se utilizan en la hipercalcemia ${ }^{2}$. En el caso descrito se utilizaron estos tratamientos saltando el escalón de los bisfosfonatos porque podían agravar la insuficiencia renal que ya presentaba la paciente; además, su eficacia está clara en la hipercalcemia maligna, pero no en otras causas de hipercalcemia ${ }^{2}$. Finalmente, y por falta de respuesta al tratamiento médico, se recurrió a la hemodiálisis como último escalón del tratamiento médico ${ }^{2}$, que en nuestra paciente se mantuvo hasta la intervención quirúrgica.
Consideramos, al igual que otros autores ${ }^{9}$, que tras estabilizar la calcemia y establecer el diagnóstico topográfico debe realizarse la paratiroidectomía precozmente, dados los buenos resultados y la curación del proceso. En este caso, debido a la falta de respuesta al tratamiento médico, se indicó la cirugía sin conseguir estabilizar a la paciente, que incluso asoció tromboembolia pulmonar que en otras situaciones contraindicaría una cirugía.

Antes de realizar la cirugía es recomendable un diagnóstico de localización mediante ecografía cervical y gammagrafía con 99Tc-sesta $\mathrm{MIBI}^{9}$, puesto que la presentación como crisis hipercalcémica se ha descrito con frecuencia en adenomas ectópicos ${ }^{10}$, y en función de la concordancia de imágenes y la asociación de patología tiroidea se indica un abordaje mediante cervicotomía central o selectivo sobre la glándula.

En esta paciente se realizó un abordaje central con hemitiroidectomía y paratiroidectomía en bloque por el gran tamaño del adenoma y la posible asociación del carcinoma de paratiroides con la crisis hipercalcémica que describen algunos autores ${ }^{7}$.

Se realizó determinación de la PTH intraoperatoria siguiendo los criterios de Miami para confirmar la curación del HPTP ${ }^{11}$ tras un descenso de su valor preexéresis > 50\% (Tabla 1). También se realizó un estudio anatomopatológico intraoperatorio para descartar la posibilidad de un falso negativo en la citología previa.

Cuando se realiza una paratiroidectomía inmediata para tratar el HPTP agudo, los pacientes pueden presentar complicaciones cardiacas dada la rápida disminución de la concentración sérica de calcio. Para prevenir esta hipocalcemia transitoria es recomendable realizar determinaciones seriadas de la calcemia y valorar la necesidad o no de administrar calcio oral e intravenoso, como hicimos en esta paciente $^{12}$.

\section{Conclusiones}

La crisis hipercalcémica por HPTP es una emergencia endocrina, pero los síntomas inespecíficos pueden retrasar su diagnóstico y su tratamiento, con un desenlace fatal. Es fundamental incluir la calcemia en cuadros clínicos inespecíficos para no retrasar el diagnóstico. El tratamiento médico intensivo permite estabilizar al paciente, pero en ocasiones es necesario el tratamiento quirúrgico urgente, que no debe demorarse si el tratamiento médico no es efectivo. El 
diagnóstico de localización preoperatorio con gammagrafía-ecografía y la determinación intraoperatoria de PTH son indispensables para conseguir un tratamiento quirúrgico curativo.

\section{Conflicto de intereses}

Los autores declaran que no existe ningún potencial conflicto de intereses relacionado con el artículo.

\section{Responsabilidades éticas}

Protección de personas y animales. Los autores declaran que para esta investigación no se han realizado experimentos en seres humanos ni en animales.

Confidencialidad de los datos. Los autores declaran que han seguido los protocolos de su centro de trabajo sobre la publicación de datos de pacientes.

Derecho a la privacidad y consentimiento informado. Los autores han obtenido el consentimiento informado de los pacientes y/o sujetos referidos en el artículo. Este documento obra en poder del autor de correspondencia.

\section{Bibliografía}

1. Cannon J, Lew JI, Solórzano CC. Parathyroidectomy for hypercalcemic crisis: 40 years experience and long-term outcomes. Surgery. 2010;148:807-13.

2. Ahmad S, Kuraganti G, Steenkamp D. Hypercalcemic crisis: a clinical review. Am J Med. 2015;128:239-45

3. Sala TD, Mureşan S, Roman R, Lazăr A, Ion R, Paşcanu I. Hypercalcaemic crisis due to primary hyperparathyroidism: report of two cases. $J$ Crit Care Med (Targu Mures). 2019;5:34-9.

4. Gómez A, García M, Barrios B, Gutiérrez MT, Gómez J, Expósito A, et al. Hiperparatiroidismo primario y crisis hipercalcémica aguda tóxica. Cir Esp. 2012;90):660-6.

5. Henzen C. Paraneoplastic endocrine syndromes. Praxis. 2018;107:1309-15.

6. Colleen K, Elizabeth $\mathrm{G}$. Surgical management of multiple endocrine neoplasia 1 and multiple endocrine neoplasia 2. Surg Clin N Am. 2019;99:693-709.

7. Gücek Haciyanli S, Acar N, Gür EO, Çelik SC, Karaıslı S, Dilek ON, et al. Severe hypercalcaemia of primary hyperparathyroidism: could giant adenoma be the real culprit rather than carcinoma? Ann R Coll Surg Engl. 2020;102:363-8.

8. Singh DN, Gupta SK, Kumari N, Krishnani N, Chand G, Mishra A, et al. Primary hyperparathyroidism presenting as hypercalcemic crisis: twenty-year experience. Indian J Endocrinol Metab. 2015;19:100-5.

9. Gómez Palacios A, Gómez Zabala J, Barrios B, Gutiérrez MT, Expósito A, Zorraquino $\mathrm{A}$, et al. Valor del diagnóstico topográfico preoperatorio en el hiperparatiroidismo (HPT). Rev Cal Asist. 2007;22:106-12.

10. Schweitzer VG, Thompson NW, Harness JK, Nishiyama RH. Management of severe hypercalcemia caused by primary hyperparathyroidism. Arch Surg. 1978;113:373-81.

11. Barczynski M, Konturek A, Hubalewska-Dydejczyk A, Cichon S, Nowak W, et al. Evaluation of Halle, Miami, Rome, and Vienna intraoperative $\mathrm{iPTH}$ assay criteria in guiding minimally invasive parathyroidectomy. Langenbecks Arch Surg. 2009;394:843-9.

12. Ameerudden $\mathrm{S}, \mathrm{He} X$. Management and surgical treatment of parathyroid crisis secondary to parathyroid tumors: report of four cases. Int Med Case Rep J. 2011;15:59-66 